\title{
Standardization of cerebrospinal fluid shunt valves in pediatric hydrocephalus: an analysis of cost, operative time, length of stay, and shunt failure
}

\author{
*Jessica Berns, BS, Blake Priddy, MD, Ahmed Belal, MBBCh, MS, PhD, R. Dianne Seibold, RN, \\ Kristin Zieles, BS, and Andrew Jea, MD, MHA \\ Section of Pediatric Neurosurgery, Department of Neurological Surgery, Indiana University School of Medicine, Indianapolis, \\ Indiana
}

\begin{abstract}
OBJECTIVE CSF shunts are the most common procedures performed in the pediatric neurosurgical population. Despite attempts in multiple studies, a superior shunt valve has never been shown. Because of this, the authors aim was to examine the impact of shunt valve standardization at their institution to determine if there is a difference in surgical cost, operative time, or short-term postoperative shunt failure.
\end{abstract}

METHODS A retrospective analysis at the authors' institution was performed for all new CSF diversion shunts, as well as shunt revisions requiring a new valve, or a new valve and at least a new proximal or distal catheter over a 1-year period (January 1, 2016, to December 31, 2016). After a period of transition, neurosurgeons were encouraged to use only one type of fixed-differential-pressure valve and one type of programmable valve when performing shunt surgeries. These patients who underwent "standardized" shunt surgery over a 1-year period (January 1, 2018, to December 31, 2018) were then compared to patients in the prestandardization epoch. All patients were followed for a 12-month period after surgery. Demographic information, surgical cost, operative time, and postoperative shunt failure data were collected in all patients in the study.

RESULTS The authors analyzed 87 shunt surgeries in patients prior to standardization and 94 shunt surgeries in patients after standardization. The rate of violation of the standardized shunt valve policy after implementation was $5.3 \%$ ( 5 of 94 procedures). When comparing the prestandardization group to those who received the standardized valve, operative costs were less $(\$ 1821.04$ vs $\$ 1333.75, p=0.0034)$. There was no difference in operative times between groups (78 minutes vs 81 minutes, $p=0.5501)$. There was no difference in total number of shunt failures between the two groups at 12 months after surgery $(p=0.0859)$. The rate of postoperative infection was consistent with the literature at $8 \%$.

CONCLUSIONS In accordance with quality improvement principles, the reduction of unexplained clinical variance invariably leads to a decrease in cost and, more importantly, increased value. In this study, the implementation of a standardized shunt valve decreased operative cost. There were no differences in postoperative shunt failures at 12 months after surgery and no differences in length of surgery. Standardizing shunt valves in the treatment of pediatric hydrocephalus seems to be cost-effective and safe.

https://thejns.org/doi/abs/10.3171/2020.8.PEDS20477

KEYWORDS shunt standardization; shunt cost; shunt operative time; pediatric hydrocephalus

$\mathrm{W}$ ITH the development of a CSF diversion shunt system in the 1950 s, the treatment of hydrocephalus was revolutionized. ${ }^{1}$ Significant advancements in shunt technology have continued to evolve. Various ventriculoperitoneal (VP) shunt valves have been created, including a wide range of fixed-differential-pressure, volume-regulated, gravity-actuated, and program- mable valves without or with in-line antisiphon devices. Several studies have tried to identify a superior shunt valve for the treatment of hydrocephalus, but none have been able to show any significant difference in shunt infection or malfunction in the large number of valves available today. ${ }^{2-7}$

Medical cost management is becoming increasingly

ABBREVIATIONS IVH = intraventricular hemorrhage; $L M C=$ lipomyelomeningocele; $M M C=$ myelomeningocele; $V A=$ ventriculoatrial; $V P=$ ventriculoperitoneal . SUBMITTED June 2, 2020. ACCEPTED August 27, 2020.

INCLUDE WHEN CITING Published online January 29, 2021; DOI: 10.3171/2020.8.PEDS20477.

* J.B. and B.P. share first authorship of this work. 
more important in today's healthcare environment. In various medical specialties, studies have shown that standardization of care is a crucial way in which cost can be decreased without sacrificing the quality of medical care provided to patients. ${ }^{8-10}$ To the best of our knowledge, however, no study has analyzed the cost-effectiveness of various shunt valves in the pediatric population.

Because prior studies have not been able to show a difference in shunt outcomes between various pediatric shunt valves, we aim to discover if creating a standardized protocol for selecting shunt valves for the treatment of pediatric hydrocephalus would have a positive cost benefit, while maintaining quality of patient care. The purpose of this study was to use our institutional experience with shunt valve standardization to show a cost benefit without extending operative time or jeopardizing patient safety.

\section{Methods}

\section{Standardization Process}

Our pediatric neurosurgical group convened to choose a single "standard" fixed-pressure valve and programmable valve. A series of weekly meetings was undertaken in person or via email until consensus was obtained. Each surgeon was given a copy of data delineating shunt valve costs. Available published studies analyzing safety, efficacy, and outcomes of shunt valve placements were reviewed. In addition, input from our nurse practitioner team was sought regarding ease of maintenance, programming of the shunt valves, and ensuring intended settings, because our nurse practitioners are typically the first point of contact for patients and families after shunt surgery.

Cost was not a factor in our decision to standardize to any particular shunt valve. That is, we did not intentionally choose the most inexpensive shunt valves in our inventory. Furthermore, it was not our intent to realize a cost savings with standardization of shunt valves. Our initiative was driven by our multisurgeon (MD) and multiprovider $(\mathrm{MD} / \mathrm{NP} / \mathrm{RN})$ practice in which we routinely cover for each other's patients. In order to do this safely and effectively, we decided to standardize our work so that we would become very familiar with the shunt apparatus used in our practice. We believed that instead of dealing with one-off, exotic shunt designs with which all of us may not be familiar, we would become very facile with management of a limited inventory of shunt valves. Our experience is not singular. Time and time again, the elimination of unexplained clinical variance leads to equivalent if not improved outcomes; as a secondary benefit, healthcare costs decrease also, thereby increasing value.

The PS Medical CSF-Flow Control Valve (Medtronic Inc.) and PS Medical StrataMR (MRI-compatible) valve (Medtronic Inc.) were selected as the preferred nonprogrammable and programmable valves, respectively, for our group. In fact, PS Medical fixed-pressure valves and Strata programmable valves were in the middle of the range of costs for comparable shunt valves. No other perioperative variables, such as draping, prepping, antibiotic use, catheter type used, or intraoperative equipment, were changed related to shunt revision surgery in our practice.

\section{Prestandardization Analysis}

A retrospective analysis was performed on all new VP and ventriculoatrial (VA) shunt placements performed at Riley Hospital for Children from January 1, 2016, to December 31, 2016, in the prestandardization epoch. In addition to all new shunts, any VP or VA shunt requiring revision of the valve alone, or of the valve and at least the proximal or distal catheter was included in the analysis. Within this time range, there were no restrictions on the type of valve or catheter used by the three pediatric neurosurgeons at our institution. The choice was solely based on surgeon preference. Data on patient age at time of surgery, sex, reason for shunt placement, type of shunt and valve, shunt cost, operative time, and need for later shunt revision within 12 months of surgery were collected for all patients who met inclusion criteria.

\section{Poststandardization Analysis}

Beginning on September 14, 2016, a new policy was developed to encourage all neurosurgeons to use only one type of nonprogrammable valve (PS Medical CSF-Flow Control Valve) and one type of programmable valve (PS Medical StrataMR) when revising or placing new shunts. There was a window of transition to a standardized valve inventory until January 1, 2018. All four surgeons agreed to and participated in this policy change. There were no restrictions on the type of catheter that could be used, or whether a programmable or nonprogrammable shunt could be placed. Data from this second poststandardization epoch from January 1, 2018, to December 31, 2018, were retrospectively analyzed. Data on patient age at time of surgery, sex, reason for shunt placement, type of shunt and valve, shunt cost, operative time, and need for later shunt revision within 12 months of surgery were collected for all patients who met inclusion criteria.

\section{Statistical Analysis}

Descriptive statistics including mean, standard deviation, standard error, and confidence interval were computed for all measurements. The $\mathrm{p}$ values were calculated using the Student t-test and chi-square test for continuous and categorical variables, respectively. Continuous variables included cost of shunt supplies and length of surgery. Categorical variables included need for shunt revision during the 12-month study period after surgery. Statistical significance was set a priori at a $\mathrm{p}$ value $<0.05$.

\section{Results \\ Patient Demographics}

Table 1 shows the demographic breakdown for patients with shunts analyzed in this study. Inclusion criteria for patients included children $<18$ years old with either first-time shunt placement or revision shunt placement necessitating valve replacement. From January 1, 2016, to December 31, 2016, 87 patients with VP, VA, or subdural peritoneal shunts met our inclusion criteria. In this prestandardization group, the average age of the patient was 6.12 years old. This group was consisted of 61 boys (70.11\%) and 26 girls $(29.89 \%)$. The most common etiology for hydroceph- 
TABLE 1. Demographics of patients undergoing new CSF shunt placement or revision of valve in the pre- and poststandardization groups

\begin{tabular}{|c|c|c|c|}
\hline Variable & Prestandardization & Poststandardization & $\mathrm{p}$ Value \\
\hline Average age (yrs) & 6.12 & 4.8 & 0.1779 \\
\hline Sex & & & 0.0195 \\
\hline Male & $61(70.11 \%)$ & $50(53.19 \%)$ & \\
\hline Female & $26(29.89 \%)$ & $44(46.81 \%)$ & \\
\hline Total & 87 & 94 & \\
\hline Etiology for hydrocephalus & & & 0.9098 \\
\hline Aqueductal stenosis & $1(1.15 \%)$ & $5(5.32 \%)$ & \\
\hline Chronic subdural & $1(1.15 \%)$ & $2(2.13 \%)$ & \\
\hline Congenital hydrocephalus & $21(24.14 \%)$ & $16(17.02 \%)$ & \\
\hline Craniosynostosis & $1(1.15 \%)$ & $2(2.13 \%)$ & \\
\hline Cyst & $5(5.75 \%)$ & $3(3.19 \%)$ & \\
\hline IVH of prematurity & $13(14.94 \%)$ & $16(17.02 \%)$ & \\
\hline MMC/LMC & $12(13.79 \%)$ & $19(20.21 \%)$ & \\
\hline Postinfectious hydrocephalus & $5(5.75 \%)$ & $4(4.26 \%)$ & \\
\hline Posttraumatic hydrocephalus & $5(5.75 \%)$ & $3(3.19 \%)$ & \\
\hline Pseudotumor & $2(2.30 \%)$ & $3(3.19 \%)$ & \\
\hline Tumor & $14(16.09 \%)$ & $13(13.83 \%)$ & \\
\hline Other (including unknown etiology) & $7(8.05 \%)$ & $8(8.51 \%)$ & \\
\hline Total & 87 & 94 & \\
\hline Type of shunt & & & 0.6326 \\
\hline Fixed-pressure valve & $63(72.41 \%)$ & $71(75.53 \%)$ & \\
\hline Programmable valve & $24(27.59 \%)$ & $23(24.47 \%)$ & \\
\hline Total & 87 & 94 & \\
\hline \multicolumn{4}{|c|}{ Indication for revision w/in 12 mos after index surgery } \\
\hline Shunt infection & $3(21.43 \%)$ & $7(28.00 \%)$ & 0.239 \\
\hline Shunt malfunction & $11(78.57 \%)$ & $18(72.00 \%)$ & 0.145 \\
\hline Total & 14 & 25 & 0.0859 \\
\hline
\end{tabular}

alus in the prestandardization group included the following: myelomeningocele $(\mathrm{MMC})$ or lipomyelomeningocele (LMC) (12 patients [13.79\%]), intraventricular hemorrhage (IVH) of prematurity (13 patients [14.94\%]), tumor-associated hydrocephalus (14 patients [16.09\%]), and congenital hydrocephalus (21 patients [24.14\%]). The prestandardization group comprised 63 fixed-differential-pressure valves (72.41\%) and 24 programmable valves (27.59\%). The prestandardization valves included proGav (Aesculap); Codman Hakim Programmable Shunt Valve, Hakim Precision Fixed Pressure Valve, and Certas Programmable Valve (Integra LifeSciences); PS Medical CSF-Flow Control Valve; and PS Medical StrataMR. Of the 87 prestandardization surgeries, $40(45.59 \%)$ were revision surgeries requiring the replacement of a new valve, or new valve and at least a new proximal or distal catheter. Of these 87 shunts, 83 were VP and 4 were VA shunts.

From January 1, 2018, to December 31, 2018, there were 94 poststandardization shunt surgeries that met our inclusion criteria. The average age of the patient in the poststandardization group was 4.8 years old. Of these, 50 patients $(53.19 \%)$ were boys and 44 patients $(46.81 \%)$ were girls. The etiology for hydrocephalus in this group included the following: IVH of prematurity (16 patients
[17.02\%]), MMC/LMC (19 patients [20.21\%]), congenital hydrocephalus (16 patients [17.02\%]), and tumor-associated hydrocephalus (13 patients [13.83\%]). Within the poststandardization group, $71(75.53 \%)$ were fixed-differential-pressure valves and $23(24.47 \%)$ were programmable valves. Of the 94 poststandardization shunt surgeries, 28 (29.79\%) were revisions meeting our inclusion criteria. Of these 94 shunts, 90 were VP and 4 were VA shunts.

\section{Comparison of Cost, Operative Time, and Outcomes}

Table 2 shows the average cost of shunt surgery, operative time, and need for revision after index surgery for patients before and after the standardization. In the prestandardization group, the average cost of the shunt surgery was $\$ 1821.04$. The prestandardization average operative time was 78 minutes, and $16.09 \%$ (14 of 87 patients) of these shunts required a later revision within 12 months of follow-up. Of the 94 shunt surgeries in the poststandardization group, 89 procedures (94.68\%) used either the prescribed PS Medical fixed-differential-pressure or Strata programmable valve. The poststandardization average cost was $\$ 1333.75$, which was significantly less than the prestandardization cost of $\$ 1821.04(\mathrm{p}=0.0034)$. The 
TABLE 2. Average cost of shunt placement or revision, average operative time, and need for later revision in all patients with shunts in the pre- and poststandardization groups

\begin{tabular}{lccc}
\hline \multicolumn{1}{c}{ Variable } & Prestandardization & Poststandardization & $p$ Value \\
\hline Average cost & $\$ 1821.04$ & $\$ 1333.75$ & 0.0034 \\
\hline Average op time (mins) & 78 & 81 & 0.5501 \\
\hline Revision rate (\%) & 16.09 & 26.60 & 0.1192 \\
\hline Revision in 12 mos due to shunt infection & 3 & 7 & 0.239 \\
\hline Revision in 12 mos due to shunt malfunction & 11 & 18 & 0.1451 \\
\hline
\end{tabular}

poststandardization average operative time was $81 \mathrm{~min}-$ utes, which was not significant when compared to the prestandardization group (79 minutes, $\mathrm{p}=0.5501$ ), and had a statistically comparable revision rate of $26.60 \%$ ( 25 of 94 patients, $\mathrm{p}=0.0859$ ). There was no significant difference in length of stay from admission date to discharge date between groups (12.80 vs 10.47 days, $\mathrm{p}=0.6706$, not shown).

The prestandardization group had 3 shunt infections (3.44\%) and 11 shunt malfunctions (12.64\%) necessitating revision within 12 months after the index surgery. The poststandardization group had a comparable incidence of shunt infections (7 patients [7.45\%]) and malfunctions (18 patients [19.15\%]) after the index surgery. There were no significant differences in postoperative shunt infection and shunt malfunction within 12 months of surgery ( $\mathrm{p}=$ 0.239 vs $p=0.145$, respectively) in the pre- and poststandardization groups.

\section{Comparison of Shunt Valve Costs}

Table 3 shows a more specific breakdown of the differences in surgical cost between the pre- and poststandardization groups with regard to fixed-differential-pressure and programmable valves. For the prestandardization group, there were a total of 87 shunt procedures: 63 were fixed-differential-pressure and 24 were programmable valves. In the 94 patients in the poststandardization group in which the standardized shunt inventory was used, 71 shunts were fixed-differential-pressure and 23 were programmable. As mentioned earlier, the average total cost of shunt components of the poststandardization group was significantly less than that of the prestandardization group. When analyzing the difference in cost between fixed-differential-pressure shunt valves, the average cost before standardization was $\$ 1074.58$ compared to $\$ 758.52$ after standardization. This decrease in cost was statistically significant $(p=0.0034)$. The cost comparison for programmable shunts showed an average prestandardization price of $\$ 3780.52$ per shunt assembly, compared to
$\$ 3005.03$ after standardization. This difference was statistically significant $(\mathrm{p}=0.0196)$.

\section{Discussion}

Since its initial development, VP shunt technology has continued to evolve. With the advent of fixed-pressure and programmable valves, neurosurgeons have attempted to identify a superior valve for the treatment of hydrocephalus, but have continually fallen short. ${ }^{2-7,11}$ To date, there has never been a study to show any significant difference in outcomes for different valves in the treatment of pediatric hydrocephalus. The shunt design trial was an international multicenter randomized trial comparing three different valves. ${ }^{5}$ The study compared standard pressure-differential valves, the Orbis Sigma valve, and the PS Medical valve in children with newly diagnosed hydrocephalus. ${ }^{5}$ This study failed to show any significant difference in shunt survival for any of the three valves. ${ }^{5}$ In a follow-up study of this same population, Kestle et al. failed to show any difference in valve outcomes at the 4-year follow-up. ${ }^{4}$ Hence, there is significant variation in the selection of a shunt valve among pediatric neurosurgeons; ${ }^{12}$ this clinical variance is not explained by scientific evidence or improved outcomes. With great uncertainty comes great opportunity; in this regard, we sought to evaluate whether the quality improvement principle of standardization could be applied to selecting CSF shunt valves.

We aimed to determine if there would be an advantage (or at least noninferiority) to a standardized workflow for shunt surgery in cost, efficiency, and outcomes, if our institution used a limited shunt valve inventory: one programmable and one fixed-differential-pressure valve option. Studies in other medical specialties have shown a benefit to operative standardization. ${ }^{8-10}$ Skarda et al. demonstrated that standardization in laparoscopic appendectomy surgical equipment resulted in decreased operative cost without a change in length of stay or postoperative complications. ${ }^{9}$

TABLE 3. Average cost of fixed or programmable shunt placement or revision, average operative time, and need for later revision in all patients with shunts in the pre- and poststandardization groups

\begin{tabular}{|c|c|c|c|c|c|c|}
\hline \multirow[b]{2}{*}{ Variable } & \multicolumn{2}{|c|}{ Prestandardization } & \multicolumn{2}{|c|}{ Posttandardization } & \multicolumn{2}{|c|}{$p$ Value } \\
\hline & Fixed & Programmable & Fixed & Programmable & Fixed & Programmable \\
\hline Average cost & $\$ 1074.58$ & $\$ 3780.52$ & $\$ 758.52$ & $\$ 3005.03$ & 0.0000016 & 0.0196 \\
\hline Average op time (mins) & 79 & 80 & 82 & 83 & \multicolumn{2}{|c|}{0.5501} \\
\hline Revision rate (\%) & 57.14 & 42.86 & 70.83 & 29.17 & \multicolumn{2}{|c|}{0.1192} \\
\hline
\end{tabular}


In that study, the six surgeons at a single institution standardized a single technique for laparoscopic appendectomy. They used one uniform doctors' preference card for this procedure, and then compared their results to historic controls. The authors found a decrease in surgical cost of more than $\$ 500.00$ per appendectomy with only a 2 -minute increase in operative time. They also found no significant difference in postoperative outcomes after transitioning to a standardized method.

Our study showed several significant results with regard to operative cost. The change in average cost for shunt components used at surgery before and after standardization of shunt valves showed a statistically significant decrease of approximately $\$ 487.29$ per procedure. When these surgeries were broken down into fixed-differentialpressure and programmable valves, the fixed-differentialpressure shunt valve cost was also less after standardization, saving more than $\$ 316.06$ per procedure. Even more dramatically, programmable shunt valves showed a reduction in cost of $\$ 775.49$ per case after standardization. These cost data may afford an opportunity and empower surgeons to partner with health systems and hospitals in gain-sharing agreements, incentivizing standardization and the elimination of unexplained clinical variance. Moreover, standardization may allow greater leverage on the part of surgeons and hospitals to obtain the best pricing from medical device companies, creating even greater possibilities for cost savings.

An important component of this analysis is understanding the impact of standardization on operative time. Standardizing a policy with less expensive material does not result in cost savings if operative time is increased and overall surgical costs increase as a result. In their study of appendectomies, Skarda et al. showed no significant change in operative time when they standardized their laparoscopic appendectomy procedure. ${ }^{9}$ Our prestandardization average operative time was 78 minutes, compared to 81 minutes after standardization-however, there was no statistically significant difference.

In addition to operative time, it is also important to ensure that postoperative complications and shunt failures are not negatively affected by standardization. In a study analyzing the impact of standardization of preoperative and postoperative care for patients undergoing colorectal surgery, Thiele et al. showed no change in postoperative complications..$^{10}$ Skarda et al. also showed no change in complications after their appendectomy standardization. ${ }^{9}$ Our study shows similar results with regard to shunt failure and revision rate. In our prestandardization group, $16.09 \%$ of the shunts required a revision during 12 months of follow-up after surgery. Our poststandardization group required revisions in $26.60 \%$ of shunts during the study period. There was no statistically significant difference in the shunt revision rate between the two groups. The literature and our results continue to support the notion that standardization of surgical procedures is safe and does not result in increased postoperative complications. Furthermore, our rate of shunt infection after the index surgery is directly in line with previous studies. ${ }^{3}$

The biggest barrier to implementation of standardized clinical work is individual bias, in terms of safety and time. ${ }^{13}$ Often, a surgeon clings to a specific technique that was taught by a mentor or may have had a singular experience with an instrument or piece of equipment that has shaped his or her decision, leading to an increased perception of patient safety. Even when there are data to support the use of specific equipment, a surgeon's personal history, stubbornness, and perception of safety can overcome these data. Cost savings often do not directly benefit the surgeon, and are often hidden from him or her, whereas a complication that may result from using unfamiliar or improperly functioning equipment directly impacts the surgeon, reducing their compliance with the standard workflow.

Feedback to and education of surgeons, perhaps in the form of scorecards, seems to be crucial to facilitating change and standardization..$^{14-16}$ Transparency of data is a powerful factor in changing the behavior of healthcare providers. Most surgeons are competitive by nature and may react to benchmarking with a striving to achieve the best outcomes, including clinical and financial outcomes. Another strategy may be to align hospital and surgeon incentives through a shared-savings program. Eiferman et al. ${ }^{17}$ showed that quality of care is not compromised by this approach, and no conflicts of interest are created.

Our study does have certain limitations. There was a difference in surgeon participation in the study. That is, the group of surgeons in the prestandardization arm was not the same group as in the poststandardization arm. This could certainly affect our operative time, length of stay, revision rates, or other outcome data. This potential bias may be ameliorated by our use of mean operative times for the pre- and poststandardization groups. A final limitation involves the follow-up available for our shunt surgeries. Because our prestandardization group underwent surgery earlier than the poststandardization group, it has more postoperative follow-up and more opportunity to develop shunt failure. However, we set 12 months of follow-up after the index procedure in both groups in an effort to normalize this bias. Longer-term follow-up may be required to provide a better assessment of this metric.

\section{Conclusions}

Standardization of CSF shunt valves at our institution for the treatment of pediatric hydrocephalus significantly decreases the operative cost of CSF shunt valves. It does not increase operative time and it does not increase postoperative shunt failure. Further research in the form of a randomized controlled study and longer follow-up would provide more definitive data on the benefits of shunt valve standardization.

\section{References}

1. Nulsen FE, Spitz EB. Treatment of hydrocephalus by direct shunt from ventricle to jugular vain. Surg Forum. 1951: 399-403.

2. Davis SE, Levy ML, McComb JG, Sposto R. The delta valve: how does its clinical performance compare with two other pressure differential valves without antisiphon control? Pediatr Neurosurg. 2000;33(2):58-63.

3. Drake JM, Kestle JR, Milner R, et al. Randomized trial of cerebrospinal fluid shunt valve design in pediatric hydrocephalus. Neurosurgery. 1998;43(2):294-305. 
4. Kestle J, Drake J, Milner R, et al. Long-term follow-up data from the Shunt Design Trial. Pediatr Neurosurg. 2000;33(5): 230-236.

5. Kestle J, Milner R, Drake J. The shunt design trial: variation in surgical experience did not influence shunt survival. Pediatr Neurosurg. 1999;30(6):283-287.

6. Robinson S, Kaufman BA, Park TS. Outcome analysis of initial neonatal shunts: does the valve make a difference? Pediatr Neurosurg. 2002;37(6):287-294.

7. Xenos C, Sgouros S, Natarajan K, et al. Influence of shunt type on ventricular volume changes in children with hydrocephalus. J Neurosurg. 2003;98(2):277-283.

8. Gurnea TP, Frye WP, Althausen PL. Operating room supply costs in orthopaedic trauma: cost containment opportunities. J Orthop Trauma. 2016;30(suppl 5):S21-S26.

9. Skarda DE, Rollins M, Andrews S, et al. One hospital, one appendectomy: the cost effectiveness of a standardized doctor's preference card. J Pediatr Surg. 2015;50(6):919-922.

10. Thiele RH, Rea KM, Turrentine FE, et al. Standardization of care: impact of an enhanced recovery protocol on length of stay, complications, and direct costs after colorectal surgery. J Am Coll Surg. 2015;220(4):430-443.

11. Lee L, King NK, Kumar D, et al. Use of programmable versus nonprogrammable shunts in the management of hydrocephalus secondary to aneurysmal subarachnoid hemorrhage: a retrospective study with cost-benefit analysis. J Neurosurg. 2014;121(4):899-903.

12. Kraemer MR, Sandoval-Garcia C, Bragg T, Iskandar BJ. Shunt-dependent hydrocephalus: management style among members of the American Society of Pediatric Neurosurgeons. J Neurosurg Pediatr. 2017;20(3):216-224.

13. Avansino JR, Goldin AB, Risley R, et al. Standardization of operative equipment reduces cost. J Pediatr Surg. 2013;48(9): 1843-1849.

14. Agarwal N, Agarwal P, Mazurkiewicz A, et al. Neurosurgical cost containment via improved physician awareness. Neurosurgery. 2016;63(CN_suppl_1):194.
15. Zygourakis $\mathrm{CC}$, Valencia V, Moriates $\mathrm{C}$, et al. A prospective controlled trial of the effect of surgeon cost scorecards on operating room surgical cost reduction. Neurosurgery. 2016; 63(CN_suppl_1):161-162.

16. Guzman MJ, Gitelis ME, Linn JG, et al. A model of cost reduction and standardization: improved cost savings while maintaining the quality of care. Dis Colon Rectum. 2015; 58(11):1104-1107.

17. Eiferman D, Bhakta A, Khan S. Implementation of a sharedsavings program for surgical supplies decreases inventory cost. Surgery. 2015;158(4):996-1002.

\section{Disclosures}

The authors report no conflict of interest concerning the materials or methods used in this study or the findings specified in this paper.

\section{Author Contributions}

Conception and design: Jea, Berns, Priddy. Acquisition of data: Berns, Priddy, Belal, Seibold, Zieles. Analysis and interpretation of data: Berns, Priddy, Belal, Seibold, Zieles. Drafting the article: Berns, Priddy, Belal, Seibold. Critically revising the article: Jea, Berns, Priddy. Reviewed submitted version of manuscript: all authors. Approved the final version of the manuscript on behalf of all authors: Jea. Statistical analysis: Jea, Berns, Priddy, Belal, Zieles. Administrative/technical/material support: all authors. Study supervision: Jea, Berns, Priddy, Seibold, Zieles.

\section{Correspondence}

Andrew Jea: Oklahoma Children's Hospital, The University of Oklahoma College of Medicine, Oklahoma City, OK. dr.andrew.jea@gmail.com. 